\title{
Star Forming Galaxies: Properties and Implications for the Extragalactic Infrared Background
}

\author{
George Helou \\ IPAC, Mail Code 100-22, California Institute of Technology, \\ 1200 E. California Blvd, Pasadena, California 91125, USA
}

\begin{abstract}
This paper reviews those properties of normal galaxies most relevant to the estimation of the extragalactic infrared background, in particular: (1) their infrared emission spectrum and its variation among galaxies; (2) their morphological properties, and their infrared size distribution in comparison to their apparent visible-light sizes; and (3) other properties such as the infrared-to-radio ratio and color-luminosity relations. Even a simple comparison of those properties to those of the extragalactic infrared background leads to constraints on the fluctuations in time and the secular evolutionary behavior of the infrared luminosity of galaxies.
\end{abstract}

\section{Introduction}

The Extragalactic Infrared Background (EIRB) arises from the superposed emission of all galaxies, integrated over all epochs back to the first such objects, however similar or dissimilar to Local Universe galaxies. Interpreting this background relies therefore heavily on our knowledge of all these galaxies. Naturally, galaxies in the Local Universe are the best studied of all, thus providing the cornerstone of this integration. While the best characterized objects are at redshifts $z \lesssim 0.1$, there is strong evidence that certain properties change little up to $z \simeq 1$, which is the range from which much of the background arises, depending on the wavelength of interest (Guiderdoni et al. 1998). Thus while the mix of objects making up the population evolves with $z$, the building blocks may well be drawn from the same set studied in the Local Universe.

This review will concentrate on the nature of the emission and the properties of normal galaxies, meaning those powered primarily by star formation. The spectral energy distributions of these galaxies, probably the most relevant property to the estimation of the EIRB, is discussed in section 2 for the midinfrared, and in section 3 for the global infrared. Section 4 addresses the infrared morphologies and sizes. Section 5 discusses the infrared-radio correlation and its relevance to the extragalactic background and deep counts.

Another significant contributor to the EIRB is the population of galaxies powered by active galactic nuclei (AGN) with various degrees of dust extinction. However, the significance of their contribution to the total EIRB is still under debate, though current estimates tend to run below 20\% (Rush et al. 1993; Heckman 1999; Mushotzky et al. 2000; Rowan-Robinson in this volume). 


\section{Mid-Infrared Spectra of Galaxies}

Until the launch of the Infrared Space Observatory, ISO (Kessler et al. 1996), the only spectral information on galaxies in the mid-infrared came from groundbased observations in the 8 to $13 \mu \mathrm{m}$ window targeting high surface brightness objects, primarily the nuclear regions of galaxies with starbursts or active nuclei (e.g., Willner et al. 1977; Roche et al. 1991). Even IRAS had detected only three or four individual galaxies with its mid-infrared spectrometer, IRAS-LRS (Cohen \& Volk 1989). ISO measured hundreds of galaxies, both at high and low spectral resolution, advancing dramatically our knowledge of this part of the spectrum.

\subsection{The Aromatic Features}

The mid-infrared spectra ( 2.5 to 5 and 5.7 to $11.6 \mu \mathrm{m}$ ) obtained by ISO-PHOT (Lemke et al. 1996) revealed the interstellar medium emission from star-forming galaxies to be strongly dominated by the aromatic features in emission (AFE) at $6.2,7.7,8.6$ and $11.3 \mu \mathrm{m}$. These appear in two main groups, one stretching from 5.5 to $9 \mu \mathrm{m}$, and the other starting at $11 \mu \mathrm{m}$ and extending beyond the spectral range of these data; see Boulanger et al. 1996 for a similar spectrum at longer wavelengths of a molecular cloud region. While there is good evidence to link these features to polycyclic aromatic hydrocarbons (PAH) or similar compounds, there is no rigorous spectral identification (Puget \& Léger 1989; Allamandola et al. 1989). It is generally agreed, however, that the emitters are small structures, $\sim 100$ atoms typically, transiently excited to high energy levels by single photons. ISO-SWS (de Graauw et al. 1996) spectra with greater spectral resolution show AFEs with the same shape, a clear indication that they are spectrally resolved by our PHT-S data.

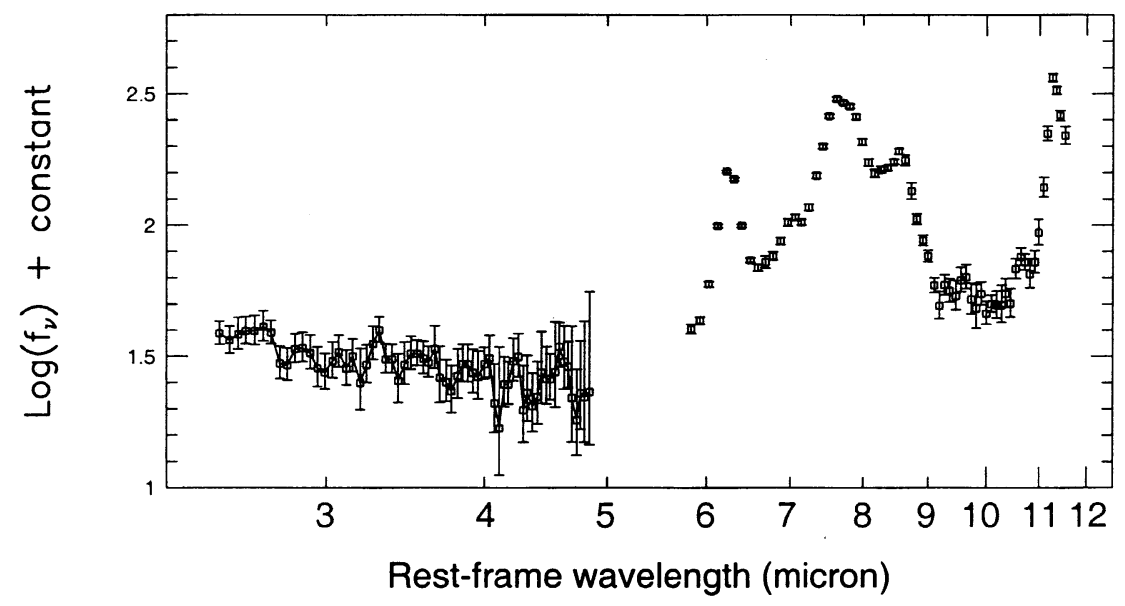

Figure 1. Composite spectrum obtained by combining data from 28 galaxies observed with ISO-PHT-S. Adapted from Helou et al. (2000).

Figure 1 shows a composite spectrum obtained from a straight average of a set of PHT-S spectra of 28 diverse star-forming galaxies taken from Helou et al. 
(2000); the individual spectra were normalized to the integrated flux between 6 and $6.6 \mu \mathrm{m}$ before averaging. The error bars, showing the dispersion among the averaged spectra, demonstrate that the mid-infrared spectral shape and relative feature strengths vary little or only weakly with galaxy attributes. Relative to each other, various feature luminosities are constant to within the signal-to-noise ratio, or $\sim 20 \%$. One exception is the relative strength of the $11.3 \mu \mathrm{m}$ feature, which varies among galaxies by as much as 40\%, (Lu et al. 1996; Lu et al. 2001). The very weak dependence of relative feature strength on galaxy parameters such as the far-infrared colors is direct evidence that the emitting particles are not in thermal equilibrium. The well known $3.3 \mu \mathrm{m}$ aromatic feature is detected at the expected wavelength, carrying about $0.5 \%$ of the power in the AFE longwards of $5 \mu \mathrm{m}$, a significantly smaller fraction than observed in M82 (Willner et al. 1977). This composite spectrum should be a reliable representation of the emission from the interstellar medium (ISM) of normal galaxies, and its properties are discussed in detail by Helou et al. (2000) and Lu et al. (2001).

Additional emission appears in between the features, and an underlying continuum is clearly evident at $3-5 \mu \mathrm{m}$, as discussed below. The features together carry 5 to $20 \%$ of the $40-120 \mu \mathrm{m}$ "FIR" (Helou et al. 1988) luminosity, with their energetic significance decreasing as the intensity of star formation and the heating in the galaxy increase. The dip at $10 \mu \mathrm{m}$ is unlikely to result from silicate absorption, since its shape is invariant among galaxies (Sturm et al. 2000).

\subsection{The 4-Micron Continuum}

A striking and unexpected aspect of the composite spectrum in Figure 1 is the smooth continuum stretching from 3 to $5 \mu \mathrm{m}$. This continuum would contribute about a third of the luminosity in the 3 to $13 \mu \mathrm{m}$ range, but only $10 \%$ between 6 and $13 \mu \mathrm{m}$, and 1 to $4 \%$ of the "FIR" luminosity. It has a $\mathrm{f}_{\nu} \propto \nu^{+0.65}$ shape between 3 and $5 \mu \mathrm{m}$, making it almost certainly of non-stellar origin, since spectra of elliptical galaxies dominated by stellar photosphere emission drop off like $f_{\nu} \propto \lambda^{-2.5} \propto \nu^{2.5}$ between 2 and $5 \mu \mathrm{m}$. The origin of this $4-\mu \mathrm{m}$ continuum cannot yet be established with certainty, but it is probably related to thermally fluctuating grains without aromatic features (Sellgren et al. 1984).

Figure 2 (Lu et al. 2001) illustrates the distinction between this continuum and the photospheric emission by comparing the spectra of spiral, ISM-rich galaxies with those of elliptical galaxies with little ISM emission. The figure shows two composite spectra, both combining mid-infrared spectra from ISO PHOT-S at $\lambda>3 \mu \mathrm{m}$ with near-infrared photometry from 2MASS at J, $\mathrm{H}$ and $\mathrm{K}$ bands. The top spectrum, displayed as squares, combines data from 17 spiral galaxies with IR/B ratios running from near 3 to more than 30 , whereas the lower spectrum, plotted as a continuous line, is derived from three elliptical galaxies all with IR/B ratios smaller than $3 \%$. The two spectra are normalized to the H-band flux. Even though the ellipticals may not be devoid of ISM emission, the ISM emission from spirals has a strong excess that increases in amplitude towards longer wavelengths starting at $3 \mu \mathrm{m}$, thus demonstrating the reality of the $4 \mu \mathrm{m}$ continuum and relating it to the ISM. See Lu et al. (2001) for a detailed analysis and discussion of these data. 


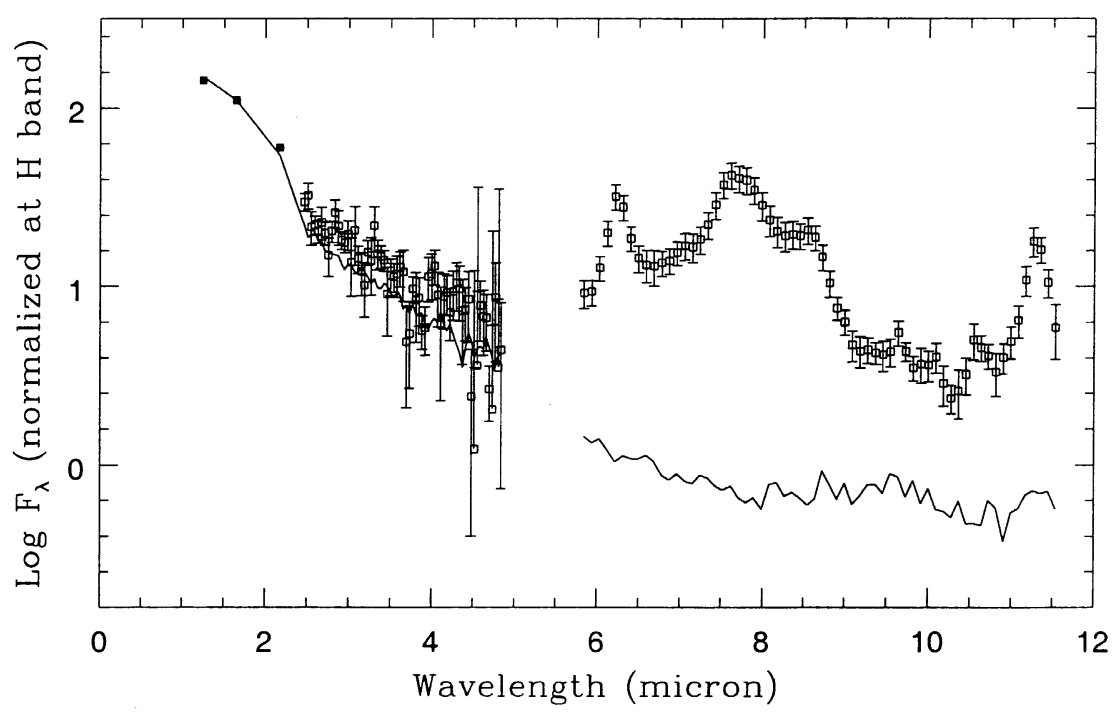

Figure 2. Comparison of data from elliptical galaxies with low infrared-to-visible ratios, shown as the line-connected dots, and spiral galaxies with high infrared-to-visible ratios, shown as the squares with error bars. The spectra are scaled to each other at the H-band data point at $1.6 \mu \mathrm{m}$. Adapted from Lu et al. (2001).

\subsection{Implications}

The spectral signatures reported here typify the integrated emission from the ISM of the majority of star-forming galaxies, and are sufficiently unique that they could be used to obtain redshifts of highly extincted galaxies up to $\mathrm{z}=3$ with the Space InfraRed Telescope Facility (SIRTF). For instance, the low-resolution mode of the InfraRed Spectrograph (IRS, Roellig et al. 1998) could readily detect $L(F I R)=10^{9} L_{\odot}$ galaxies out to $z=0.1$ in about an hour of integration time, whereas ultraluminous galaxies may be detectable out to $z=3$ in a comparable amount of time depending on the AFE-to-far-infrared ratio assumed (Weedman, private communication).

For galaxies with known redshifts, AFE detection would be an unmistakable dust signature, and thus instantly distinguish between thermal and nonthermal mid-infrared emission, and quantify their relative importance (Genzel \& Césarsky 2000). AFE may also be used to estimate the amount of extinction in high-redshift systems, or otherwise constrain ISM parameters, and thus improve the star formation rate estimates.

As the redshift of galaxies increases, the AFE emission will move with respect to any band used for extragalactic surveys. As each emission feature passes in and out of the observing band, the galaxy will become easier then harder to detect. For instance, galaxies at redshifts near one were particularly favored for detection in the $15 \mu \mathrm{m}$ filter in the ISOCAM (Césarsky et al. 1996) camera, because that placed the strong $7.7 \mu \mathrm{m}$ feature optimally in the $15 \mu \mathrm{m}$ band. Thus, $15 \mu \mathrm{m}$ surveys towards clusters turned up an enhanced number count around 
a redshift of 1 (Barvainis et al. 1999; Altieri et al. 1999). The same effect makes also for structure in source count curves as a function of flux density in single-band surveys. The differential source counts at wavelengths between 6 and $24 \mu \mathrm{m}$, for instance, will show "wiggles", then turn over and drop quickly once the AFE are redshifted beyond the filter wavelength (Xu et al. 1998).

\section{Global Infrared Spectra of Galaxies}

One of the most important attributes of the EIRB is its spectral shape, which provides tests of models, and constrains the epoch of production of the radiation. The interpretation of this spectral shape however is severely hampered without a reliable representation of the spectra of the galaxies that collectively make up the background (e.g., Guiderdoni et al. 1998). IRAS data have been a unique and major source of information, but they do not constrain the spectral shapes sufficiently for the detailed modeling needed. Still, they indicate unambiguously that single or double black-body curves, grey or modified by an emissivity dependence on wavelength are not an adequate representation of the infrared spectra of galaxies (Helou 1989). ISO provided spectro-photometry in the midinfrared ( $\S 2$ above), and in the far-infrared for the brightest sources, as well as photometry in a variety of new bands between 3 and $200 \mu \mathrm{m}$, thus enabling a more accurate understanding of the spectral energy distributions (SED), and more reliable modeling as described in this section.

\subsection{Sorting Out the Colors of Dust Emission}

It was clear from the earliest IRAS data that the SEDs of galaxies follow a main sequence of shapes. At the quiescent end, the SED is similar to the emission from the quiescent cool to warm ISM, whereas the active end is dominated by emission from the star-forming, high density and high intensity ISM (Helou 1986). Figure 3 shows the IRAS evidence for the sequence, and adds the ISO perspective as the ISO-IRAS color diagram (Dale et al. 2000). These color diagrams are direct evidence that the SED sequence is coherent across the whole range from a few $\mu \mathrm{m}$ to longwards of $100 \mu \mathrm{m}$.

Besides describing accurately the SED family for galaxies, it is important to characterize the parameters that are most efficient at sorting that family. While it is often assumed that the color sequence corresponds to a luminosity sequence, Figure 4 demonstrates that not to be the case. The left-most panel in Figure 4 is equivalent to the right-hand panel in Figure 3, with the axes switched around. The right-most panel shows that luminosity is not nearly as efficient at predicting the mid-infrared color as the far-infrared color. Dale et al. (2001) discuss the sorting statistics of SEDs, and conclude that $R(60 / 100)=f_{\nu}(60 \mu \mathrm{m}) / f_{\nu}(100 \mu \mathrm{m})$ is the best sorting index, meaning it is the first order parameter in placing a galaxy on the family of SED shapes. Luminosity is clearly not an effective sorting index, at least for the sample used by Dale et al (2001); if luminosity fails for this representative sample, it is unlikely to work in more global samples which include this one as a subset.

An interesting parallel to this analysis is presented by Shibai et al. (2000) and Okumura et al. (2000), who combine IRTS data at $155 \mu \mathrm{m}$ with IRAS data to demonstrate that SEDs of HII regions in the Milky Way depend primarily 


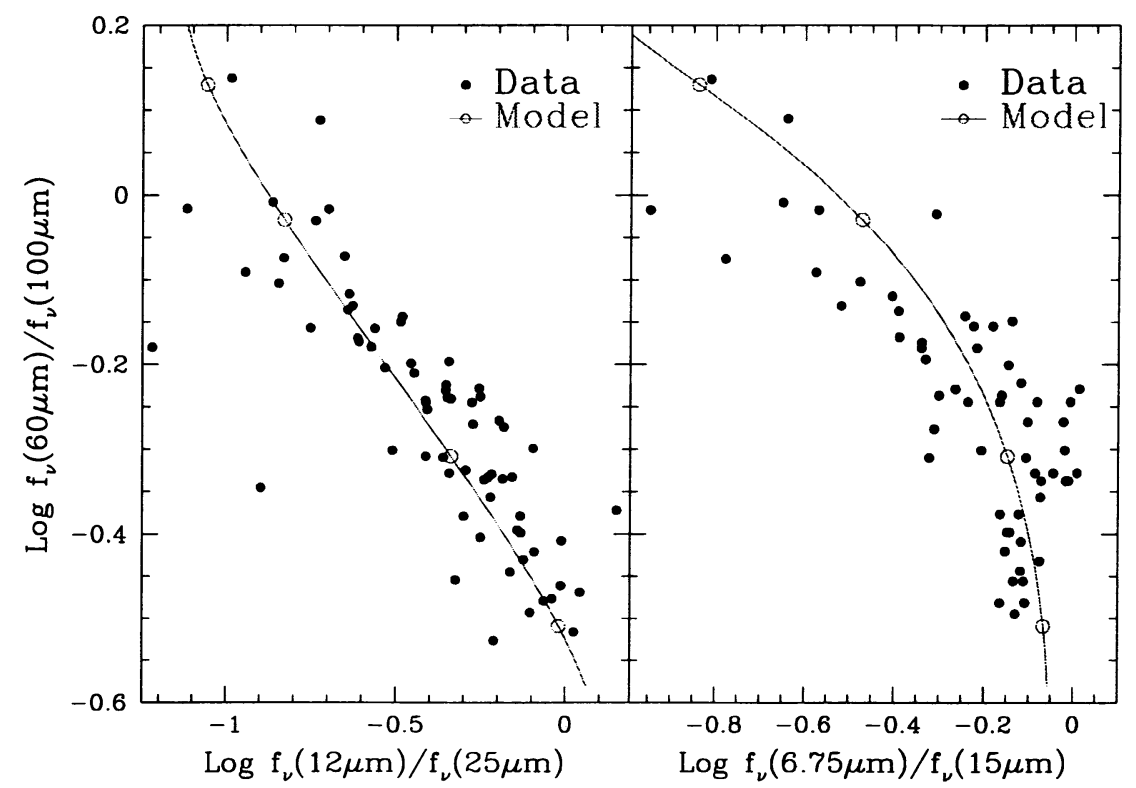

Figure 3. Color-color diagrams from IRAS data (frame to the left), and from ISO and IRAS data (frame to the right). The common ordinate can be taken as indicative of the intensity of dust heating in each galaxy. The lines running through the data represent the locus of the synthetic SED models described in $\S 3.2$.

on the local estimated heating intensity. They then examine the correlations between the various emitting dust components and conclude that fluctuations are still important at $60 \mu \mathrm{m}$.

Using that first-order parameter result, Dale et al. (2001) derived the mean SED for each range of $R(60 / 100)$, as shown in Figure 5. This figure combines IRAS and ISO data, for about sixty galaxies taken from the ISO Key Project on Normal Galaxies (Helou et al. 2000). The spectra are all normalized to $60 \mu \mathrm{m}$, so the dispersion in the other bands is indicative of the efficacy of using $R(60 / 100)$ as the key parameter in describing the SED. This plot can be read as the combination of all the color-color diagrams similar to Figure 3 that can be constructed from the photometric data in the six bands shown. Note for instance three systematic changes taking place as the heating and $R(60 / 100)$ increase: The peak emission shifts towards shorter wavelengths, the ratio of $15 \mu \mathrm{m}$ to $7 \mu \mathrm{m}$ emission rises, and the fractional contribution of the mid-infrared to the total emission decreases.

The middle frame in Figure 4 has intermediate scatter between the left and right frames. This probably points to a second-order parameter in sorting SEDs, which can be broadly associated with the mid-IR to far-IR flux ratio. Such a second-order parameter might be hard to discern in the data because of the systematic trend of decreasing mid-IR relative to far-IR as $R(60 / 100)$ increases. 


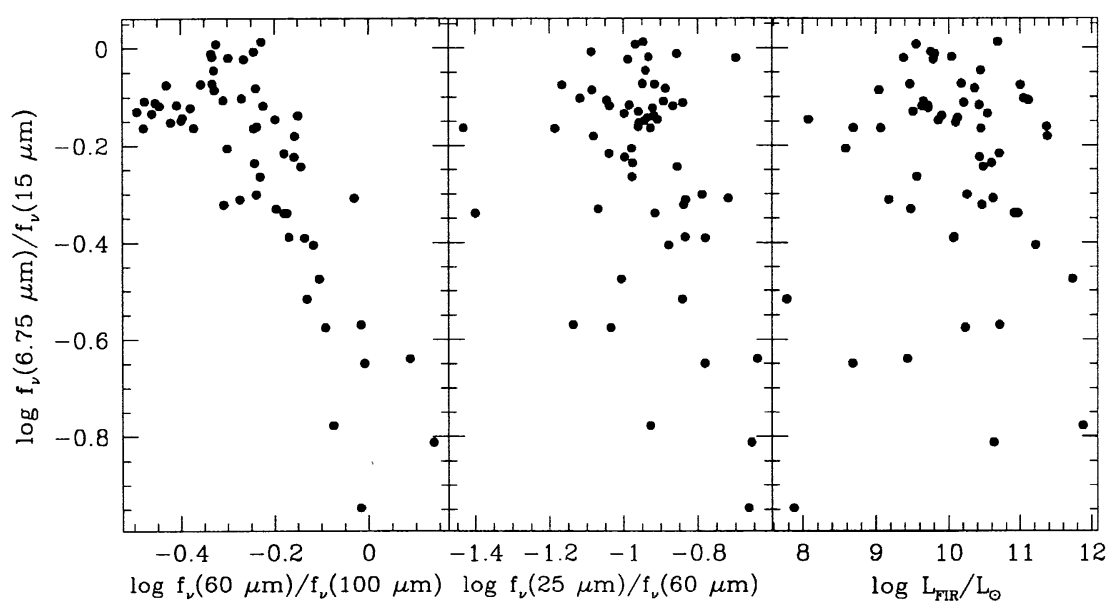

Figure 4. Scatter diagrams of the ISOCAM 6.75 to $15 \mu \mathrm{m}$ ratio against each of three other quantities in the ISO Key Project sample of galaxies. The cleaner correlation in the left-most frame argues for $\mathrm{R}(60 / 100)$ being the "best" parameter to use in describing the SED of a galaxy. Adapted from Dale et al. (2001).

\subsection{Synthetic Spectra of Galaxies}

Guided by the empirical evidence in the preceding section, one could construct analytical descriptions of the SEDs. The limited data from IRAS provided incomplete constraints on such analytical models, so much of the modeling used component-based approaches, describing the SEDs as linear combinations of fixed components with variable coefficients to generate the SED sequence (Helou 1986; Rowan-Robinson 2000). Other authors even resorted to combinations of blackbody curves to model the mid-infrared emission, which is known to be far from thermal equilibrium. Examples of recent efforts at modeling the farinfrared to submm SEDs can be found in Siebenmorgen et al. (1999), Calzetti et al. (2000), and Bekki \& Shioya (2000).

The data however continue to become richer with additions obtained from ISO and ground-based submillimeter telescopes, and are anticipated to become richer still as data become available from SIRTF and other future missions such as IRIS, FIRST and Planck. These improved constraints necessitate more realistic models which take into account in particular the natural spread in heating environments encountered in each galaxy studied in spatial detail. Dale et al. (2001) offer such a new-generation model, which describes each galaxy as harboring a power-law distribution of dust mass $M_{d}$ over interstellar radiation fields $U$ spanning from 0.3 to 1000 times the field intensity in the solar neighborhood:

$$
d M_{d}(U) \propto U^{-\alpha} d U, \quad 0.3 \leq U \leq 10^{3}
$$

For each radiation field intensity, an emission curve is constructed which includes contributions from large grains in thermal equilibrium, very small grains with fluctuating effective temperature, and aromatic feature carriers (e.g., Désert, 


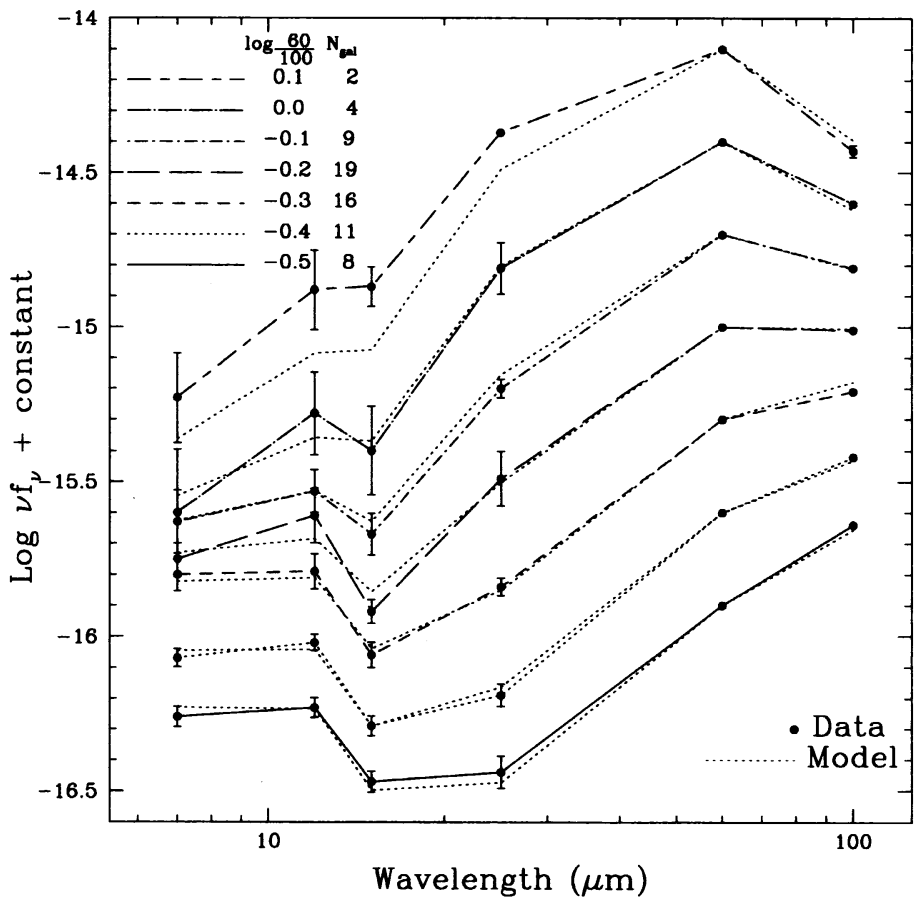

Figure 5. The empirical spectra of galaxies sorted on the far-infrared color $\mathrm{R}(60 / 100)$, shown as filled circles connected by heavy lines. The thinner lines show the equivalent data simulated from the synthetic SED models described in $\S 3.2$. Adapted from Dale et al. (2001).

Boulanger, \& Puget 1990). Achieving a best fit to the data constrains the relative importance and spectral shapes of these components as discussed by Dale et al. (2001). In this implementation of the model, the range of heating intensities is kept fixed, and the only variable is $\alpha$.

This single-parameter model coincides well with the single-parameter empirical sequence of the SEDs, with the $R(60 / 100)$ color mapping to the power-law index of the distribution. The index $\alpha$ ranges from 2.5 for the least active galaxy to just over 1 for the most active, inviting the question of its physical significance. The value of $\alpha$ can in fact be related to the structure of the dusty ISM. In the case of a diffuse medium where the heating intensity falls off primarily because of $r^{-2}$ dimming, one would write $d U / d r \propto-r^{-3}$. For a uniform medium with $d M_{\mathrm{d}} \propto r^{2} d r$, the relation $d M_{\mathrm{d}} \propto U^{-2.5} d U$ obtains. On the other hand, in a dense medium where the heating intensity is primarily attenuated by dust absorption, one would write $d U / d r \propto-U$ for a slab geometry. For a uniform slab, $d M_{\mathrm{d}} \propto d r$, so $d M_{\mathrm{d}} \propto U^{-1} d U$ obtains. Thus, $\alpha \sim 2.5$ is a reasonable approximation for the diffuse cirrus-like components of the ISM, whereas $\alpha \sim 1$ is a good approximation for a photodissociation region near young stars. A variable $\alpha$ parameter may therefore be interpreted as the varying contributions of active and quiescent regions from galaxy to galaxy. 


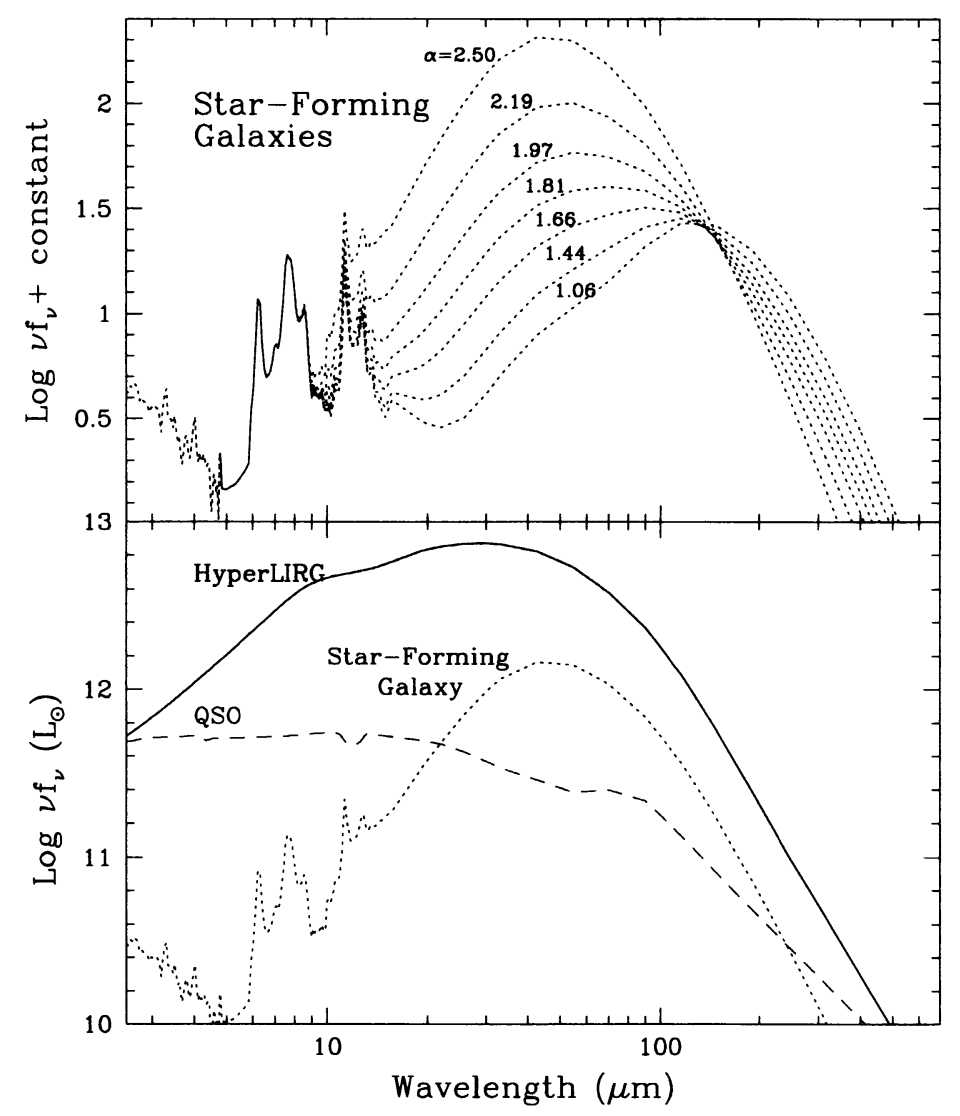

Figure 6. The fully detailed synthetic SED models derived by Dale et al. (2001) are shown in the upper frame. For comparison, the lower frame illustrates two other SED shapes associated with galaxies powered by a dust-enshrouded AGN (labelled HyperLIRG) and by a nearly unextincted quasar.

The resulting SEDs from Dale et al. (2001) are shown in Figure 6 as a sequence of spectra closely matching the empirical sequence of Figure 5. In Figure 6 , the spectra are normalized to the AFE at $6.2 \mu \mathrm{m}$ for a clearer display. One can readily see here the main trends identified in Figure 5. Another expression of the models is shown in Table 1, which tracks the broad-band energy distribution as a function of $\alpha$. The Dale et al. (2001) model reproduces well the empirical spectra in Figure 5, where the model output is plotted as the broken lines. Similarly, the mean trends in infrared colors shown in Figure 3 are reproduced quite well by the model, with the remaining scatter presumably due to measurement errors as well as unmodelled fluctuations about the main SED sequence. It should be pointed out that these SED models are not matched to any data at $\lambda \geq 200 \mu \mathrm{m}$, and need to be better constrained there. 
Table 1. Infrared Energy Budget

\begin{tabular}{cccccccc}
\hline$R(60 / 100)$ & $\alpha$ & $3-5 \mu \mathrm{m}$ & $5-13 \mu \mathrm{m}$ & $13-20 \mu \mathrm{m}$ & $20-42 \mu \mathrm{m}$ & $42-122 \mu \mathrm{m}$ & $122-1100 \mu \mathrm{m}$ \\
& & $\%$ & \multicolumn{1}{c}{$\%$} & $\%$ & $\%$ & $\%$ & $\%$ \\
\hline 0.31 & 2.50 & 2.6 & 13.1 & 3.3 & 7 & 35 & 39 \\
0.40 & 2.19 & 2.3 & 11.9 & 3.4 & 10 & 41 & 32 \\
0.51 & 1.97 & 2.0 & 10.2 & 3.5 & 14 & 46 & 24 \\
0.63 & 1.81 & 1.6 & 8.5 & 3.7 & 18 & 50 & 19 \\
0.79 & 1.66 & 1.2 & 6.8 & 4.0 & 22 & 52 & 14 \\
1.00 & 1.44 & 0.8 & 4.9 & 4.4 & 28 & 54 & 9 \\
1.32 & 1.06 & 0.4 & 3.3 & 4.9 & 34 & 53 & 5 \\
\hline \hline
\end{tabular}

\subsection{Applications}

Establishing an accurate SED model has several applications which are closely connected to the EIRB, besides its direct estimation. One of those is placing the flux measured for a given object in a given band in the context of the global SED, especially when this is the only measurement available, and an interpretation is needed without knowledge of $R(60 / 100)$ (Calzetti et al.2000). Table 1 suggests, for instance, that the mid-infrared flux in the band 13 to $20 \mu \mathrm{m}$ is a good indicator of the total infrared emission, in agreement with arguments by Roussel et al. (2001). It also suggests that the 20 to $40 \mu \mathrm{m}$ range carries a fraction of the total infrared luminosity which rises steeply with increasing $R(60 / 100)$, at the expense of the submm fraction of the luminosity. These two bands in particular will significantly bias surveys in the sense of favoring certain types of objects at equal total infrared luminosity, and will be the most difficult to scale up to a total luminosity absent evidence about the galaxy's infrared colors.

A commonly debated question (Calzetti et al. 2000) concerns the ratio of the total infrared flux to the "FIR" flux derived originally from $f_{\nu}(60 \mu \mathrm{m})$ and $f_{\nu}(100 \mu \mathrm{m})$ measured by IRAS (Helou et al. 1988). Figure 7 plots the value of that ratio derived from the SED models described above as a function of redshift, assuming that the FIR measuring filters are held fixed in the observer's frame. The most noticeable effect is that the correction factor increases quickly on average beyond $z=0$, growing by a factor of several by $z \gtrless 3$. The range covered by the possible values of this correction factor also widens dramatically, translating into an uncertainty in the factor to apply without other information on the infrared colors. Thus, while "FIR" is a good indicator at $z=0$ of the total infrared flux even in the absence of color information, it degrades quickly as redshift increases.

The third application illustrated here is the use of SED models to predict the color diagrams of galaxies at high redshifts. Figure 8 shows two colorcolor diagrams expected for different combinations of four bands accessible with SIRTF. The smooth solid line is the locus at $z=0$ expected for normal galaxies. Each circle on that line stands for one of the models shown in Table 1. As redshift increases from 0 to 1 in steps of 0.1 , these circles are expected to move 


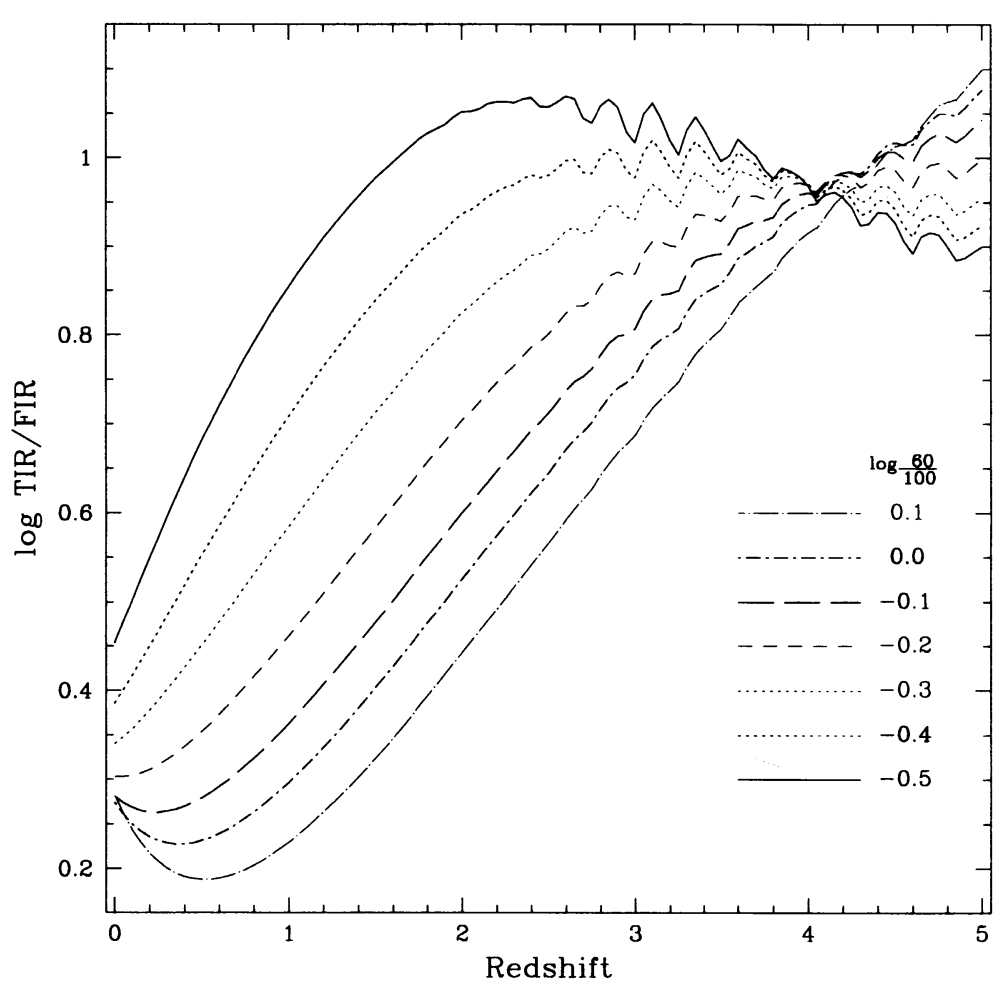

Figure 7. The expected variation in the total to "FIR" flux as a function of redshift of the emitting galaxy, using the Dale et al. (2001) synthetic SED models. TIR denotes the total dust luminosity, integrated from 3 to 1100 microns. Each curve corresponds to one of the model spectra shown in the upper panel of Figure 6.

along the broken lines as shown. One can readily see in the right-hand frame of Figure 8 that near the middle of the color sequence, the colors plotted are of little value in estimating redshift. In the left-hand frame however, objects always move away from the $z=0$ line, and degeneracy in interpretation sets in only at redshifts greater than a half.

\subsection{Discussion}

In summary, the infrared SEDs of galaxies are well described to first order as a single-parameter family. The parameter that best sorts the SED sequence appears to be $R(60 / 100)$. In order to describe the full SED from the UV to the submillimeter, one needs at least one additional parameter, namely the infraredto-blue ratio or an equivalent quantity. While this ratio is broadly correlated with $R(60 / 100)$, the two are not equivalent. For example, a galaxy containing a small but intense star formation burst will have warm infrared colors but a low infrared-to-blue ratio.

It is important to note that while the SEDs described above apply to the majority of star-forming galaxies, they do not reproduce all spectra of galaxies. 


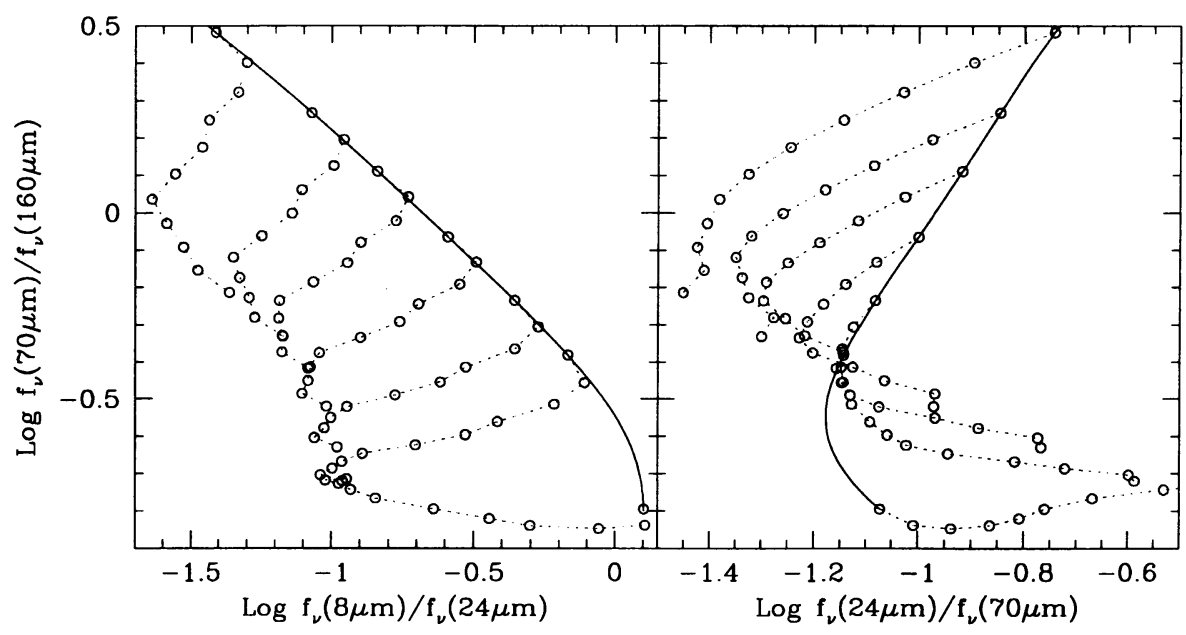

Figure 8. The expected range in SIRTF colors at $z=0$ (solid curve in each frame), and expected variation in SIRTF colors as a function of redshift of the emitting galaxy, using the Dale et al. (2001) synthetic SED models. Each broken line traces the change in color of the same galaxy as its redshift increased from 0 to 1 , with the sequence of open circles diverging from the parent point on the $z=0$ curve in steps of $z=0.1$.

For instance, Ultra-Luminous Infrared Galaxies tend to have steeper spectra in the mid-infrared, suggesting that the spectrum is modified by significant optical depth in the emitting dust. Though rare, these objects are important because they will be the highest redshift population picked up in deep surveys. Another such instance are the galaxies powered predominantly by an AGN, whose spectra display completely different properties in the mid-infrared, and are much flatter at all wavelengths, due either to dust heated by very intense radiation, or to direct non-thermal emission from the nucleus. Hyperluminous galaxies and QSOs are extreme examples of this case, as shown in Figure 6. This class of AGN-dominated galaxies is thought to provide 10 to $20 \%$ of the EIRB, a fraction that should be accurately established by SIRTF.

Perhaps most significant is that spectra of low-metallicity galaxies seem to be substantially different from the normal galaxy spectra described above. The best known example may be SBS0335-052, whose mid-infrared spectrum appears to peak shortwards of $20 \mu \mathrm{m}$, and have much weaker AFE (Thuan et al.1999). The importance of this observation is that metallicity must decrease with increasing redshift, so that local Universe model SEDs may become increasingly poorer representations of the actual spectra that contribute to the EIRB and provide the faintest sources detected in infrared surveys. Here again, SIRTF will help gauge the redshift at which low metallicity becomes a significant factor affecting the SEDs. 


\section{Infrared Morphology of Galaxies}

The spatial structure of the EIRB depends on the internal structure of the contributing galaxies and on their clustering properties. While near-term missions like SIRTF or FIRST will not have sufficient spatial resolution to detect the EIRB fluctuations due to internal galaxy structure, future missions like NGST might. Morphological studies of star forming galaxies are also instrumental in deriving a proper relation between the infrared flux and the star formation rate (Kennicutt 1998; Malhotra et al. 1996; Roussel et al. 1999), which is in turn critical to the interpretation of the EIRB.

\subsection{Mid-Infrared Structure}

Dale et al. (2000) presented data and analysis for sixty-one galaxies selected for the ISO Key Project on Normal Galaxies (Helou et al. 2000), and observed at 6.75 and $15 \mu \mathrm{m}$ with ISOCAM. These galaxies have optical diameters mostly in the one to a few arcminute range, but include larger galaxies like IC 10, NGC 1313 and NGC 6946. Roussel et al. (2000) presented another forty-three galaxies observed under the Guaranteed Time of the ISOCAM team, and with optical sizes larger than the Dale et al. compilation, including well known and well resolved galaxies such as M 101, M 51, NGC 1365, NGC 4569, NGC 5236, and NGC 6744. These and other data revealed that many of the general features of galaxies associated with their visible appearance, such as spiral arms, HII complexes and rings, are also seen in the mid-infrared. The most significant exception is that prominent bulges and disks dominated by old stars fade away in the infrared (Madden et al. 1999). All images show strong structure down to the resolution limit of the ISOCAM maps, namely about 7 arcsec, even in the most nearby galaxies like M 101. Most disk galaxies that are active in star formation tend to have their activity concentrated in a central brightness enhancement (Dale et al.2000). The maps are generally similar at 6.75 and $15 \mu \mathrm{m}$, except in galaxies with a nuclear star-burst, where the latter tends to be more prominent at $15 \mu \mathrm{m}$ than at $6.75 \mu \mathrm{m}$. This is consistent with the color trends shown in Figure 3, where the ratio of $6.75 \mu \mathrm{m}$ to $15 \mu \mathrm{m}$ is depressed in more active regions. Roussel et al. (1999) present evidence for a greater frequency among early-type galaxies of nuclear enhancement against the disk emission, and discuss the role of bars in causing these enhancements. This is consistent with results based on IRAS data that early-type galaxies, including lenticulars, tend to have lower infrared-to-blue ratios than late-type spirals, but also tend on average to have warmer far-infrared colors, which are typical of star-bursts (Sauvage \& Thuan 1994).

\subsection{Mid-Infrared Sizes}

Dale et al. (2000) compared sizes of galaxies in the mid-infrared and in the visible. Fitting elliptical isophotes to the mid-infrared images, they found that the mean brightness profiles fall off exponentially with radius, down to $\sim 0.2$ $\mathrm{MJy} \mathrm{sr}^{-1}$. Extrapolating the observed profiles slightly yields an estimate that the mid-infrared sizes match the blue light size $R_{25}$ at surface brightnesses $B_{\nu}(6.75 \mu \mathrm{m}) \simeq 0.04 \mathrm{MJy} \mathrm{sr}^{-1}$ and $B_{\nu}(15 \mu \mathrm{m}) \simeq 0.09 \mathrm{MJy} \mathrm{sr}^{-1}$, with uncertainties of about $25 \%$, but with a scatter among galaxies close to $50 \%$. This result 
and more details of the comparison are shown in Figure 9, which is taken from Dale et al. (2000).

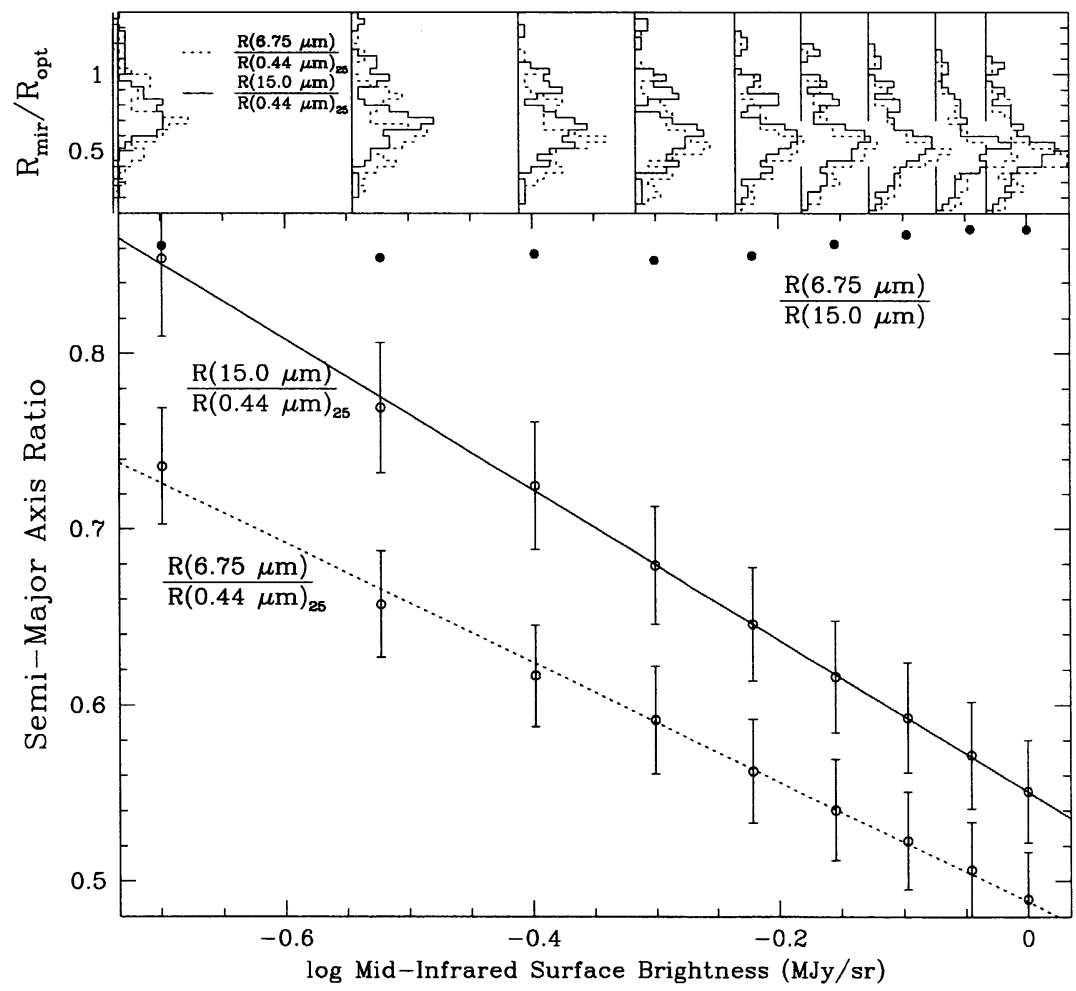

Figure 9. The mid-infrared sizes at various values of surface brightness, normalized to $R_{25}$ measured at $25 \mathrm{mag} \operatorname{arcsec}^{-2}$ in the B-band (lower frame). The upper frame shows the histograms of values that enter the plotted mean ratios. Adapted from Dale et al. (2000).

The mean surface brightnesses in the mid-infrared found by Dale et al. coincide in amplitude with the estimate by Boulanger \& Pérault (1988) and in color with the estimate by Reach \& Boulanger (1998) for the face-on brightness of the Milky Way in the solar neighborhood. Dale et al. conclude that the mean emission in the outskirts of normal galaxy disks are indeed similar to cirrus emission in the Milky Way, and that these outer disks appear to have a ratio of total infrared cooling to total heating input of order 0.7 , accurate to within a factor 2 .

\section{The Radio-IR Relation and the Extragalactic Radio Background}

The tightest and most universal correlation known among galaxy fluxes connects the far-infrared emission from dust heated by stars with the radio emission in the 2 to $50 \mathrm{~cm}$ range, which is dominated by synchrotron radiation from cosmic ray electrons trapped in the interstellar magnetic field. The infrared-to-radio 
luminosity ratio is about $5 \times 10^{5}$. Condon (1992) and Helou (1991) have reviewed empirical evidence and theoretical work in this area. While the correlation is established in the Local Universe at $z \lesssim 0.01$, Hacking et al. (1989) found that even in a sample of galaxies with a median redshift of 0.1 , the infraredto-radio ratio maintained the "canonical" Local Universe mean and dispersion. IRAS F10214 +4724 at $z=2.3$ has been shown to follow the correlation with an abnormally low infrared flux, though the significance of that fact is diluted by the questions surrounding the nature of the power source in this object (RowanRobinson et al. 1993; Clements et al. 1993). More recent studies, especially those involving submillimeter deep surveys, have generally added to the evidence that the correlation holds to $z \sim 1$ (Richards 1999), and have encouraged the use of high-resolution radio continuum maps to locate submillimeter sources (Barger et al. 2000).

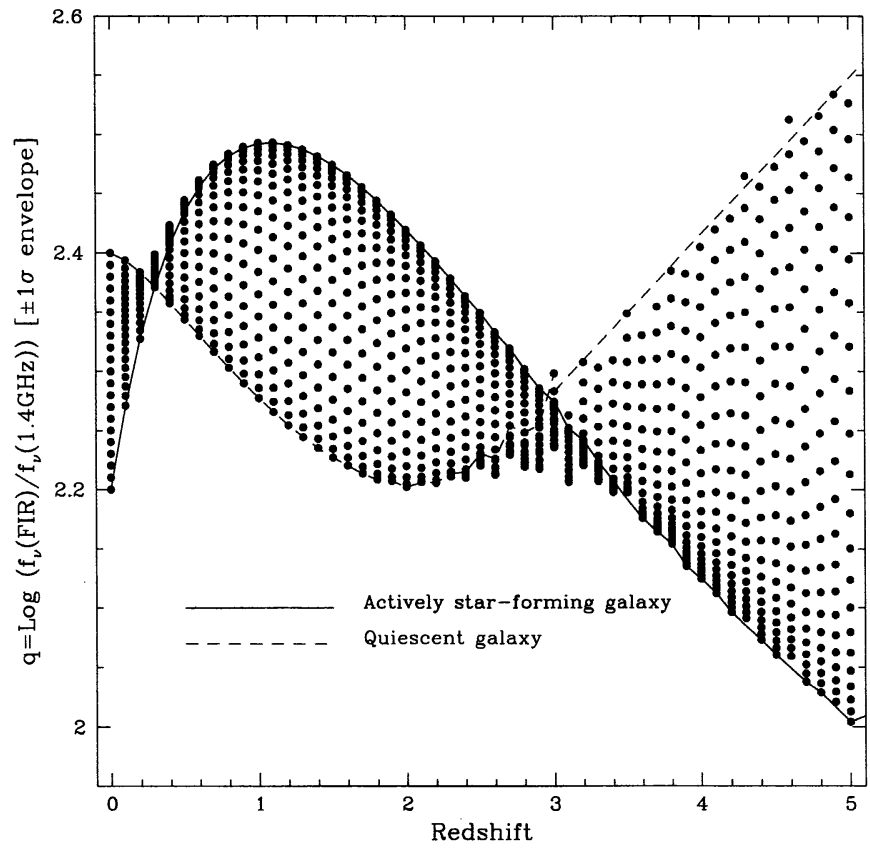

Figure 10. The variation in the infrared-to-radio ratio as a function of redshift of the emitting galaxy, estimated in the observer's rest frame. Adapted from Dale et al. (2001).

Assuming the infrared-to-radio range of ratios holds independent of redshift, Figure 10 shows the expected values of that ratio as a function of the redshift of the targeted population. The ratio $Q$ is defined as in Helou, Soifer, \& RowanRobinson (1985) to be the ratio of flux density in the far-infrared (flux FIR over a bandwidth of $3.7510^{12} \mathrm{~Hz}$ ) to flux density at $1.4 \mathrm{GHz}$, and we use the notation $\mathrm{q}=\log (\mathrm{Q})$. Condon et al. (1991) show that the $\pm 1 \sigma$ range of $\mathrm{q}$ among galaxies is $\sim 0.2$ wide, and that more actively star-forming galaxies, i.e., those with higher infrared-to-blue ratios, tend to lower values of q. Helou (2000a, §3.4) associates the properties of more active galaxies with a younger stellar population, and 
by extension generally warmer dust. Using those arguments, one can assign the warmer SEDs ( $§ 3)$ to the lower value of $q$, and therefore map the range of synthetic SEDs to the $\pm 1 \sigma$ range of observed q, and compute the estimates for Figure 10. Interestingly, the observed ratio does not change significantly with redshift, even though the range is "inverted" at $z \simeq 0.2$ and again at $z \simeq 3$.

Given that the infrared-radio correlation holds out to $z \simeq 2$, one implication is that an extragalactic radio background would be expected, and can be estimated by scaling from the EIRB. Using the estimates in Figure 10 and assuming that the background is dominated by galaxies at redshifts up to $z \sim 2$, one gets an estimate for the radio background of order $3 \times 10^{3} \mathrm{kJy} \mathrm{sr}^{-1}$ scaling directly from the observed power in the EIRB of about $20 \mathrm{nW} \mathrm{m} \mathrm{m}^{-2} \mathrm{sr}^{-1}$. This is about half the observed total extragalactic radio background (Haarsma \& Partridge 1998), which is consistent with the other half being due to active galactic nuclei, radio galaxies and quasars (Condon 1989).

Acknowledgments. I would like to thank Danny Dale for help with the figures, and Nanyao Lu for sharing material prior to publication. This work was supported by ISO data analysis funding from the U.S. National Aeronautics and Space Administration, and carried out at the Infrared Processing and Analysis Center and the Jet Propulsion Laboratory of the California Institute of Technology.

\section{References}

Allamandola, L. J., et al. 1989, ApJS, 71, 733

Altieri, B., et al. 1999, A\&A, 343, L65

Barger, A. J., et al. 2000, AJ, 119, 2092

Barvainis, R., Antonucci, R., \& Helou, G. 1999, AJ, 118, 645

Bekki, K., \& Shioya, Y. 2000, ApJ, 542, 201

Boulanger, F., \& Pérault, M. 1988, ApJ, 330, 964

Boulanger, F., et al. 1996, A\&A, 315, L325

Calzetti, D., et al. 2000, ApJ, 533, 682

Césarsky, C., et al. 1996, A\&A, 315, L32

Clements, D. L., et al. 1993, MNRAS, 262, 23

Cohen, M., \& Volk, K. 1989, AJ, 98, 1563

Condon, J. J. 1989, ApJ, 338, 13

Condon, J. J., Anderson, M.L., \& Helou, G. 1991, ApJ, 376, 95

Condon, J. J. 1992, ARA\&A, 30, 575

Dale, D. A., et al. 2000, AJ, 120, 583

Dale, D. A., et al. 2001, ApJ, in press

de Graauw, T., et al. 1996, A\&A, 315, L49

Désert, F. X., Boulanger, F., \& Puget, J. L. 1990, A\&A, 237, 215

Genzel, R., \& Cesarsky, C. 2000, ARA\&A, 38, 761

Guiderdoni, B., et al. 1998, MNRAS, 295, 877

Haarsma, D. B., \& Partridge, R. B. 1998, ApJ, 503, L5. 
Hacking, P., et al. 1989, AJ, 339, 12

Heckman, T. M. 1999, Ap\&SS, 266, 3

Helou, G. 1986, ApJ, 311, L33

Helou, G., et al. 1988, ApJS, 68, 151

Helou, G. 1989, in Proceedings of IAU Symposium 135, Interstellar Dust, ed. L. J. Allamandola \& A. G. G. M. Tielens (Dordrecht: Kluwer), 285

Helou, G. 1991, in ASP Conf. Ser. Vol. 18, The Interpretation of Modern Synthesis Observations of Galaxies, ed. N. Duric \& P. Crane (San Francisco: ASP), 125

Helou, G., et al. 2000, ApJ, 532, L21

Helou, G. 2000a, in Les Houches Summer School LXX, Infrared Astronomy: Today and Tomorrow, ed. F. Casoli, J. Lequeux, \& F. David (SpringerVerlag: Les Ulis), 337.

Kessler, M. F., et al. 1996, A\&A, 315, L27

Kennicutt, R. 1998, ApJ, 498, 541

Lemke, D., et al. 1996, A\&A, 315, L64.

$\mathrm{Lu}, \mathrm{N}$, et al. 1996, BAAS, 28, 1356

$\mathrm{Lu}, \mathrm{N}$., et al. 2001, in preparation

Madden, S., et al. 1999, in ESA SP-427, The Universe as Seen by ISO, ed. P. Cox \& M. F. Kessler (Noordwijk: ESA/ESTEC), 933

Malhotra, S., et al. 1996, A\&A, 315, L161

Mushotzky, R., et al. 2000, Nature, 404, 459

Okumura, K., et al. 2000, in Star Formation 1999, ed. T. Nakamoto (Nagoya: Nobeyama Radio Observatory)

Puget, J.-L., \& Léger, A. 1989, ARA\&A, 27, 161

Reach, W. T., \& Boulanger, F. 1998, in The Local Bubble and Beyond, ed. D. Breitschwerdt, M. J. Freyberg, \& J. Truemper (Berlin: Springer-Verlag)

Richards, E. 1999, ApJ, 513, L9

Roche, P. F., et al. 1991, MNRAS, 248, 606

Roellig, T. L., Houck, J. R., Van Cleve, J., et al. 1998, SPIE, 3354, 1192

Roussel, H., et al. 1999, in ESA SP-427, The Universe as Seen by ISO, ed. P. Cox, \& M. F. Kessler (Noordwijk: ESA/ESTEC), 957

Roussel, H., Vigroux, L., Bosma, A., et al. 2000, A\&A, submitted

Roussel, H., Sauvage, M., \& Vigroux, L. 2001, in preparation

Rowan-Robinson, M., et al. 1993, MNRAS, 261, 513

Rowan-Robinson, M., et al. 2000, MNRAS, 316, 885

Rush, B., et al. 1993, ApJS, 99, 1

Sauvage, M., \& Thuan, T. X. 1994, ApJ, 429, 153

Sellgren, K. 1984, ApJ, 277, 623

Shibai, H., Okumura, K., \& Onaka, T. 2000, in Star Formation 1999, ed. T.

Nakamoto (Nagoya: Nobeyama Radio Observatory)

Siebenmorgan, R., Krügel, E., \& Chini, R. 1999, A\&A, 351, 495 
Sturm, E., et al. 2000, A\&A, 358, 481

Thuan, T. X., et al. 1999, ApJ, 516, 783

Willner, S., et al. 1977, ApJ, 217, L121

Xu, C., Hacking, P. B., Fang, F., et al. 1998, ApJ, 508, 576

\section{Discussion}

Michael Rowan-Robinson: I wanted to ask about your comment that the $10 \mu \mathrm{m}$ silicate feature is not seen. Surely it must come in as you move to higher luminosities?

George Helou: This feature does not appear as part of the SED sequence, so that warm far-infrared colors are not sufficient to introduce it. It does not appear in the archetypal star bursts M 82 or NGC 253, as discussed by Sturm et al. in a recent paper. Among star-forming galaxies, it seems to show up only when the galaxy is ultra-luminous, possibly because the total gas column in the system is high enough to start having layering effects, with the colder outer shells absorbing emission from the inner warmer dust.

Daniele Pierini: How does the classification of the IR SEDs correlate with the optical/near-IR colors and the $\mathrm{H}-\alpha$ equivalent widths of the late-type galaxies?

Helou: We have not tried to make that correlation with these data. To first order however, the correlations must be the same ones observed between those quantities and the 60 -to- $100 \mu \mathrm{m}$ color ratio.

H. Shibai: The physical meaning of the $60 / 100$ micron ratio is not clear. We have already executed a similar analysis by using the $100 / 150$ micron ratio for the Galactic plane. The result is very clear.

Helou: I agree that the $60 \mu \mathrm{m}$ band of IRAS has been shown to be affected by fluctuating grains, so that it is not as clean an indicator of heating intensity as if it were exclusively filled by large grains in equilibrium. However, 60 and $100 \mu \mathrm{m}$ data are readily available for thousands of galaxies from the IRAS survey, so they are a practical choice rather than the optimal choice in the most general case. 